Serial cholangiograms from one patient
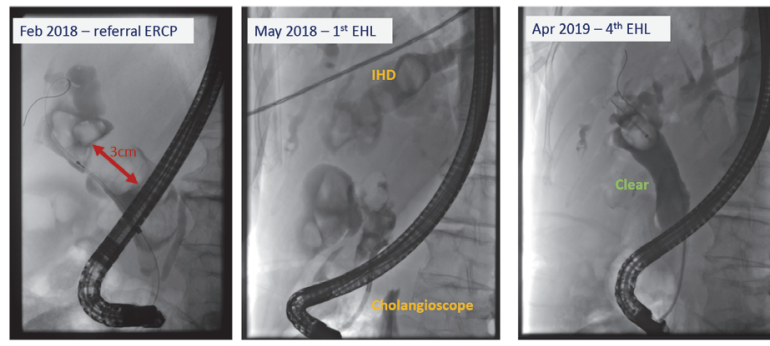

Abstract PTU-50 Figure 1 Serial cholangiograms from one patiet

discharged from A\&E. $80 \%$ of these discharged patients had an Oakland Score >8. $21(17.2 \%)$ of admitted patients received an inpatient lower GI endoscopy; $19 \%$ of which received endoscopic therapy. The most commonly identified cause of LGIB was diverticulosis (23.8\%). Overall, $82.8 \%$ of admitted patients received no inpatient lower GI endoscopy and were managed conservatively. Comparing those who underwent LGI endoscopy versus a watch and wait approach, there was no difference in inpatient mortality $(0 \%$ vs $4 \%$, $\mathrm{p}=1.0)$ or 30 -day re-admission rate $(9.5 \%$ vs $22.8 \%, \mathrm{p}=$ 0.24). However, undergoing inpatient LGI endoscopy was associated with greater median length of stay (8 days vs 3 days, $\mathrm{p}=0.0002$ )

Conclusions Age and co-morbidities complicate risk stratification in the elderly as many will score highly regardless of bleed severity, limiting the role of the Oakland Score. Endoscopic assessment of all elderly patients presenting with LGIB is not performed in real world practice, may not be necessary or even appropriate. Although not in keeping with current guidelines, this watch and wait approach does not appear to be associated with adverse outcomes in the elderly.

\section{PTU-51 PROSPECTIVE OBSERVATIONAL COHORT VALIDITY STUDY VIRTUAL REALITY ENDOSCOPIC SIMULATION}

${ }^{1}$ Catherine Eley*, ${ }^{2}$ Neil Hawkes, ${ }^{3,4}$ Richard Egan, ${ }^{1}$ David Robinson, ${ }^{1,3}$ Chris Brown, ${ }^{1}$ Wyn Lewis. 'Health Education And Improvement Wales, Cardiff, UK; ${ }^{2}$ Royal Glamorgan Hospital, Cardiff, UKi ${ }^{3}$ Morriston Hospital, Swansea, UK; ${ }^{4}$ Swansea University, Swansea, UK

\subsection{6/gutjnl-2021-BSG.124}

Introduction The benefit of simulated endoscopy training is inversely proportional to trainee experience. The aim of this study was to determine the face validity of the EndoSim highfidelity virtual reality simulator (Surgical Science, Gothenburg, Sweden). and establish benchmark metric values to inform research into endoscopy skill acquisition, learning curve trajectory, and curriculum development.

Methods A pilot cohort of four experts rated simulated exercises by Likert-scale (1-5). Following iterative development, 10 experts completed a 13-exercise simulator-based curriculum, with 35 individual amounting to 858 total metric values. Statistical analysis for non-parametric data was used: where multiple comparisons were made, Bonferroni calculation was performed which altered the standard significance of $\mathrm{p}<0.05$ to $\mathrm{p}<0.0014$.

Results There was no significant difference in expert performance in any metric across all exercises $(p>0.0014)$. Face validity was determined by expert Likert score ratings (1-5 [1: very poor, 5: very good]) and varied between exercises (median Likert-scale score 4 [3-5). Lower GI exercises: Loop Management and Intubation Case 3, had worse face validity (median Likert-scores 3: [IQR 1-3, and 2-3 respectively]) compared to basic handling exercises: Scope Handling, Visualise Colon 1 and 2 (median scores 4.5: [IQR 3-5, 4-5 and 4-5 respectively]).

Conclusions Overall, experts felt the EndoSim curriculum had good face validity for basic scope handling skill acquisition: the next focus will be in establishing translation of these skills into clinical practice and as such, its' future role in Endoscopy training.

\section{PTU-52 SURVEILLANCE ENDOSCOPY FOR BARRETT'S OESOPHAGUS}

Athina Mavrou*, Sadek Malas, Evgenia Kolyvaki, Mathew Vithayathil, Sophie Stevens, Jonathan Hoare, Anet Soubieres. Imperial College Healthcare Nhs Trust, London, UK

\subsection{6/gutjnl-2021-BSG.125}

Introduction Correct endoscopic evaluation of a possible Barrett's segment is essential for correct diagnosis and avoidance of unnecessary, sometimes lifelong surveillance procedures.

The British society of Gastroenterology guidelines define Barrett's oesophagus as:

'an oesophagus in which any portion of the normal distal squamous epithelial lining has been replaced by metaplastic columnar epithelium, which is clearly visible endoscopically $(\geq 1 \mathrm{~cm})$ above the GOJ and confirmed histopathologically from oesophageal biopsies' Histopathological confirmation is based on the presence of intestinal metaplasia.

In cases of short segment Barrett's $(<3 \mathrm{~cm})$ accurate endoscopic assessment can be difficult.

Our project aimed to determine what proportion of patients with short segment Barrett's oesophagus at Imperial College Healthcare NHS Trust, were correctly endoscopically diagnosed, and thus, what proportion may be undergoing unnecessary surveillance procedures.

Methods A retrospective cohort study was performed, identifying cases of short segment Barrett's $(<3 \mathrm{~cm})$ at Imperial NHS Trust between 2017 and 2020. Imaging was reviewed by an independent consultant endoscopist to determine whether endoscopic evaluation criteria had been met.

Results 261 cases of short segment Barrett's were identified. 25 had no available imaging and 10 patients were under surveillance following HALO/EMR or surgery for oesophageal cancer.

Of the remaining 226 cases, 97 had been booked for surveillance, 37 were discharged and 92 were neither discharged nor booked for surveillance.

Of the 97 already booked, 43 (44\%) were felt not to meet endoscopic criteria. Of the 92 not yet booked, 49 (53\%) did not meet the criteria.

Of the 37 patient discharged, 4 were felt to meet endoscopic criteria and had intestinal metaplasia on histology. Of these, 2 were over the age of 80,1 moved out of area and 1 was lost to follow up.

Removing cases which did not meet endoscopic criteria for surveillance, would give a $44 \%$ reduction in unnecessary gastroscopies for patients who had already been booked. Including those who had not yet been booked, this would give a $49 \%$ reduction overall. 
Conclusions Short segment Barrett's can be difficult to assess accurately. In our analysis, cases that did not meet the criteria for Barrett's diagnosis on endoscopy, had segment $<1 \mathrm{~cm}$ or an atypical $\mathrm{Z}$ line.

We have shown a potential $49 \%$ reduction in unnecessary gastroscopies, which could result in avoidance of unnecessary health and procedural anxiety for patients, and cut waiting lists and associated costs.

In addition, we suggest that patients with previous suspected Barrett's should be evaluated on dedicated surveillance lists, by endoscopists with expertise in Barrett's assessment.

\section{PTU-53 EFFICACY AND SAFETY OF ENDOSCOPIC AMPULLECTOMY IN THE UK}

Sujith Sasidharan Nair*, Mohamed Abdelrahim, Lazaros Varytimiadis, Asma Al-Kandari, Patrick Goggin, Professor Pradeep Bhandari. Queen Alexandra Hospital,Portsmouth Hospital University Trust, Portsmouth, UK

\subsection{6/gutjnl-2021-BSG.126}

Introduction Endoscopic ampullectomy is a minimally invasive technique of treating non-invasive lesions involving the ampulla of Vater and offers an alternative to major surgical intervention. In this study, we describe the safety and outcome of this procedure from a single large tertiary unit in the UK.

Methods Data were prospectively collected on an electronic database. Parameters related to ampullectomy outcome and complications were retrospectively analyzed.

10 to $20 \mathrm{~mm}$ snare sizes were used in these patients. ENDOCUT Q effect 2 was used. All patients were given indomethacin suppositories. Submucosal injection was performed only where the ampullary lesions spilled over to the duodenal mucosa.

Results A total of 48 cases were included in this analysis, between 2009 and 2021. The mean age was 62.4 years. Female represented $55.3 \%$ of patients. Mean duration of follow up was 118.29 weeks (27 months). 29 (60.4\%) lesions were pure ampullary, and the remaining involved the duodenal wall as well. Lesions ranged from 5 to $80 \mathrm{~mm}$ in size, with average size of $22.2 \mathrm{~mm}$. 6 patients (12.5\%) had familial adenomatous polyposis FAP. Pre resection histology confirmed HGD in one patient (2\%), and neuro-endocrine tumor NET in one case (2\%). Post resection histology showed focal adenocarcinoma in 2 patients (4.1\%).

Prophylactic PD stenting was performed in 31 cases $(64.5 \%)$ of which one patient developed pancreatitis. Out of the 17 cases (35.4\%) who did not have PD stenting, none developed pancreatitis. Adjunctive APC used in 11 cases (22.9\%), and submucosal injection in 28 cases $(58.3 \%)$. Enbloc resection was achieved in $16(33.3 \%)$, while piecemeal in 32 cases $(66.6 \%)$. Recurrence observed in 12 out of 48 cases (25.0\%). Piecemeal resection was significantly associated with higher risk of recurrence $(\mathrm{P}=0.0404)$. Early bleeding (including intra-procedural and bleeding within 24 hours of procedure) happened in 6 patients $(12.5 \%)$, delayed bleeding (after 24 hours) in 2 cases (4.1\%), all were successfully treated endoscopically. Only 1 patient (2\%) had Pancreatitis, and one patient developed ampullary stenosis post procedure. There was no report of perforation, need for emergency surgery or 30-day mortality in this series.

Conclusion

- Endoscopic ampullectomy seems safe and effective in expert hands
- The recurrence rate observed in this series was $25 \%$, all of them treated with further endoscopic therapy

- Piecemeal resection was significantly associated with higher risk of recurrence, all managed with further endoscopic therapy

- If indomethacin suppositories are universally used, prophylactic pancreatic stenting may not be needed as the risk of pancreatitis is very low

\section{PTU-54 EARLY PROOF OF CONCEPT STUDY OF A NOVEL ULTRASONIC MEASUREMENT DEVICE FOR UPPER GI ENDOSCOPY}

${ }^{1,2}$ Israa Bondoqa*, ${ }^{1}$ Simon Toh, ${ }^{2}$ Raymond Lee, ${ }^{2}$ Martino Pani. ${ }^{1}$ Portsmouth Nhs Trust, Dept of Upper GI Surgery, Portsmouth, UK; ${ }^{2}$ University of Portsmouth, Faculty of Technology, Portsmouth, UK

\subsection{6/gutjnl-2021-BSG.127}

Introduction Measurement of the distance of an endoscope tip from mouth guard is estimated visually in the dark by the endoscopist. To improve the precision and ease of measurement, we developed a prototype device that attaches to any endoscope externally so that this measurement can be displayed on-screen in real-time for recording and review.

Methods A prototype ultrasonic measurement device that clamps to the endoscope outside the patient was devised and tested by an experienced and novice endoscopist with multiple repeated measurements made of a fixed $Z$ line at $39 \mathrm{~cm}$. Paired measurements were made with the endoscopist's own done by usual visual inspection. The device measures how much of the endoscope is outside the patient to calculate the distance of the scope tip within the patient. This study was performed in a Koken EGD simulator (GTSimluators, Davie, Florida, USA)

Results Fifteen paired measurements each were performed by both endoscopists with the results summarized in this table (Table 1.):

There were no significant differences in the measurements made by the device compared with either endoscopists. The device did not impede endoscopy in any way, The initial study guided improvements in the design and data processing to reduce variability.

Conclusions This early proof of concept feasibility study has encouraged us to develop a workable prototype device that we will test in a series of upper GI endoscopies in patients (ScopeMeasure Study IRAS Ref 20/SC/0387). This device will be compatible with any endoscope and will be reusable within a disposable sterile sleeve. We hope that automated

\begin{tabular}{llll} 
Abstract PTU-54 Table 1 & & \\
\hline & $\begin{array}{l}\text { Mean (Range) } \\
\mathrm{cm}\end{array}$ & $\begin{array}{l}\text { Median (1SD) } \\
\mathrm{cm}\end{array}$ & $\begin{array}{l}\text { Paired student t-test (2 } \\
\text { tailed) }\end{array}$ \\
\hline $\begin{array}{l}\text { Experienced endoscopist } \\
\text { results }\end{array}$ & $39.1(39-39.5)$ & 39 & $\mathrm{p}=0.183$ \\
$\begin{array}{l}\text { Device Results } \\
\text { Experienced endoscopist } \\
\text { results }\end{array}$ & $\begin{array}{l}30.1(37-46) \\
\text { Device Results }\end{array}$ & $39(2.5)$ & \\
\hline
\end{tabular}

